Published in final edited form as:

Vaccine. 2019 July 09; 37(30): 4055-4060. doi:10.1016/j.vaccine.2019.05.081.

\title{
Safety of Guidelines Recommending Live Attenuated Influenza Vaccine for Routine Use in Children and Adolescents with Asthma
}

\author{
James D. Nordin, MD, MPH, Gabriela Vazquez-Benitez, PhD, Avalow Olsen, BS, Leslie C. \\ Kuckler, MPH, Ashley Y. Gao, Elyse O. Kharbanda, MD,MPH \\ HealthPartners Institute, Minneapolis, MN
}

\begin{abstract}
Objective: Evaluate whether a guideline recommending Live Attenuated Influenza Vaccine (LAIV) for children 2 years and older with asthma increased risks for lower respiratory events (LREs), within 21 or 42 days of vaccination, as compared to standard guidelines to administer Inactivated Influenza Vaccine (IIV) in children with asthma.
\end{abstract}

Methods: This was a pre/post guideline retrospective cohort study of children ages 2-17 years with asthma and receiving one or more influenza vaccines in two large medical groups from 20072016. Both groups recommended IIV in the pre-period; in 2010, one group implemented a guideline recommending LAIV for all children, including those with asthma. Main outcomes were medically attended LREs within 21 and 42 days after influenza immunization. Analysis used a generalized estimating equation regression to estimate the ratio of rate ratios (RORs) comparing pre/post events between LAIV guideline and control group.

Results: The cohort included 7851 influenza vaccinations in 4771 children with asthma. Among patients in the LAIV guideline group, the proportion receiving LAIV increased from $23 \%$ to $68 \%$ post-guideline implementation, versus an increase from 7 to $11 \%$ in the control group. Age and baseline asthma severity adjusted ROR showed no increase in LREs, primarily asthma exacerbations, following implementation of the LAIV guideline: overall aROR (95\% Confidence Interval): 0.74 (0.43-1.29) for LRE within 21 days of vaccination, $0.77(0.53-1.14)$ for LRE within 42 days of vaccination. For the subset of children ages 2-4 years aROR: 0.92 (0.34-2.53)

\footnotetext{
Corresponding author Contact Information: James D. Nordin, MD, MPH, James.D.Nordin@HealthPartners.Com, Senior Research Investigator, HealthPartners Institute, Mail Stop 23301A, $817033^{\text {rd }}$ Ave South, Minneapolis, MN 55425, 952-967-5087 . Contributors' Page

James Nordin conceptualized and designed the study, interpreted the data, drafted the initial manuscript, revised the manuscript critically for important intellectual content, and approved the final manuscript as submitted.

Gabriela Vazquez-Benitez designed the study, analyzed and interpreted the data, reviewed and revised the manuscript critically for important intellectual content, and approved the final manuscript as submitted.

Avalow Olsen contributed to data acquisition and interpretation of the data, and reviewed and revised the manuscript, and approved the final manuscript as drafted.

Leslie Kuckler contributed to study design, data acquisition, reviewed and revised the manuscript, and approved the final manuscript as drafted.

Ashley Gao participated in interpretation of the data, and reviewed and revised the manuscript, and approved the final manuscript as drafted.

Elyse Kharbanda conceptualized and designed the study, interpreted the data, revised the manuscript critically for important intellectual content, and approved the final manuscript as submitted.

Financial Disclosure: None to report
} 
for LRE within 21 days of vaccination and 0.94 (0.49-1.82) for LRE within 42 days of vaccination; for children 5-18 years aROR (95\% CI): 0.58 (0.26-1.30) for LRE within 21 days of vaccination and $0.67(0.37-1.23)$ for LRE within 42 days.

Conclusion: In a large cohort of children with asthma, a guideline recommending LAIV rather than IIV did not increase LREs following vaccination.

\section{Keywords}

Vaccine Safety; Asthma; Pediatrics; Immunization Guidelines; Influenza Vaccine

\section{Introduction}

Each year in the United States, among children younger than 18 years, there are an estimated 9.4 million influenza cases, resulting in 7.4 million outpatient visits and more than 300,000 hospital days. ${ }^{1}$ Children and adolescents with asthma are at increased risk for influenzaassociated morbidity. Although the prevalence of pediatric asthma is $9 \%$, children with asthma account for at least one-third of pediatric influenza hospitalizations. ${ }^{2}$ Thus, children with asthma have long been a priority group for influenza vaccination efforts. ${ }^{3,4}$

Since 2003, two influenza vaccine formulations have been available in the United States: the inactivated influenza vaccine (IIV), administered intramuscularly, and the live attenuated influenza vaccine (LAIV), administered intranasally. Results from clinical trials conducted prior to the $2009 \mathrm{H} 1 \mathrm{~N} 1$ pandemic demonstrated LAIV to have increased efficacy in children over IIV for prevention of culture-confirmed influenza. ${ }^{5-7}$ For several years, LAIV was recommended by the Advisory Committee on Immunization Practices (ACIP) preferentially over IIV for otherwise healthy children and adolescents. At the time, asthma and wheezing were listed as a precaution both in the package inserts and the ACIP recommendations. Both IIV and LAIV are now recommended by ACIP without preference for the 2018-19 influenza season. ${ }^{8}$ However, wheezing in children less than 5 years of age is listed as a contraindication and asthma in children older than age 5 as a precaution because two studies showed increased risk of wheezing in young children receiving LAIV as compared to IIV. 5,9 Other trials failed to detect increased risks following LAIV. 6,7,10,11 To date, post-marketing observational studies have not identified significant increased risks for wheezing or other lower respiratory complications following LAIV. These studies have shown protective effects, although these may have been due to confounding by indication. In these observational studies patients receiving LAIV had less severe asthma than those given IIV. 12,13

This study is based on the adoption of different guidelines for LAIV in two medical groups. Starting in the 2010-11 influenza season, one large medical group, embedded within an integrated health system, implemented a clinical guideline recommending LAIV preferentially over IIV for all pediatric patients, including those with asthma or a history of wheezing. Another large group in the same health system continued recommending IIV for children with asthma or recurrent wheezing. The goal of this study was to evaluate the safety of the LAIV guideline with a specific focus on risks for lower respiratory adverse events, following LAIV in children and adolescents with asthma or history of recurrent wheezing. 


\section{Methods}

\section{Overview}

We used a retrospective observational cohort using a pre-post controlled design ${ }^{14}$ to evaluate the safety of a clinical guideline to administer LAIV preferentially over IIV for children and adolescents with asthma or history of recurrent wheezing. Outcomes were medically attended lower respiratory events (LREs), primarily wheezing, and hospitalization within 21 and 42 days of vaccination. This study was approved by the HealthPartners Institutional Review Board with a waiver of informed consent.

\section{Setting}

Data from this observational study came from two large similar medical groups serving demographically similar populations primarily in Minnesota, operating under independent clinical leadership within a Minnesota-based nonprofit integrated health care system. The LAIV guideline medical group switched its preference for all children to LAIV in 2010 and included 21 primary care clinics. The control medical group continued using IIV for children with asthma throughout the study period and has 26 clinics. For the 2007-08 through 2009-10 influenza seasons, both medical groups recommended IIV for all children and adolescents with asthma, consistent with national guidelines at the time. ${ }^{15}$ Beginning with the 2010-11 influenza season, the LAIV medical group implemented a clinical guideline recommending LAIV preferentially over IIV for all pediatric patients, including those with asthma or a history of wheezing. In contrast, the standard of care for the control group for the 2010-11 through 2014-15 influenza seasons was to follow ACIP guidelines ${ }^{4}$ and recommend IIV for patients with asthma and for children 2 through 4 years of age with a history of wheezing.

\section{Study population}

We included children and adolescents 2 through 17 years of age during one or more influenza seasons from 2007-08 through 2014-15 with diagnoses of asthma or recurrent wheezing and who received one or more influenza vaccines. To be included for a given influenza season, subjects were required to have continuous insurance enrollment for the period from one year prior to immunization to 42 days after the immunization and have at least one primary care or asthma related subspecialty encounter (allergy, family medicine, internal medicine, pediatrics, pulmonary medicine or urgent care). Asthma was defined as having at least two asthma diagnoses on different days (ICD-9-CM codes 493.xx) at outpatient, emergency department (ED), or inpatient visits, in the 12 months prior to influenza vaccination. Children 2 through 4 years old with recurrent wheezing were required to have at least two diagnoses on 2 separate days of 786.07 (wheezing), 786.09 (respiratory distress), 466.x (bronchiolitis), or asthma (493.xx) in the 12 months prior to influenza vaccination. This definition was used consistently in both groups in both pre- and postperiods.

We excluded subjects with contraindications for LAIV including pregnancy, conditions associated with immune deficiency, malignancy, cystic fibrosis, other chronic lung disease, neuromuscular disease, organ transplant, kidney failure or autoimmune disease, enteritis and 
colitis, rheumatoid arthritis and associated diseases, identified through ICD-9-CM codes in medical record or administrative data within one year prior to influenza immunization. We also excluded subjects with any hospitalization or ED visit or an outpatient encounter for an LRE in the 42 days before influenza vaccination to avoid counting continuation of care encounters. See figure 1.

Subjects could be included in more than one influenza season if they remained eligible based on the above inclusion and exclusion criteria.

\section{Exposure}

Data from the 2007-08 through 2009-10 influenza seasons were included in the preguideline period, and data from the 2010-11 through 2014-15 influenza seasons were included in the post-guideline period. All children meeting inclusion criteria, regardless of the influenza vaccine received, were analyzed in the system in which they received the vaccine. Influenza vaccines administered within the two medical groups were identified from electronic health record-based immunization registries. For those receiving two doses of the influenza vaccine in a single season, only the first dose was included.

\section{Outcomes}

Acute, medically attended lower respiratory events (LREs), were identified from inpatient, emergency, or outpatient visits through the following ICD-9-CM codes: 466.x (acute bronchiolitis), 490 (bronchitis), 518.81, 518.82, 518.84 (acute respiratory failure), 519.11 (acute bronchospasm), and 786.00, .05-.07, .09, .2, .52, and .59 (wheezing). Inpatient or emergency visits for asthma (493.x) were included.

In addition, asthma diagnoses occurring in the outpatient setting were included when classified as exacerbations by meeting the following criteria: ICD9-codes: 493.x1 (asthma with status asthmaticus) OR 493.x2 (asthma with exacerbation) OR 786.07 (wheezing) for children less than 5 years, OR nebulizer treatment CPT 94640, OR prescription for oral corticosteroid. This algorithm was validated using chart abstraction as the gold standard (algorithm sensitivity 67\% (95\% CI: 54-80\%), specificity 90\% (95\% CI: 81-99\%)). ${ }^{16}$ All urgent care and ED visits and hospital admissions with asthma diagnosis codes were considered exacerbations.

Predefined post-vaccination risk windows for outpatient visits were 1-21 days and 1-42 days; for inpatient or emergency visits, risk windows were 0-21 days and 0-42 days, consistent with previous research. ${ }^{17,18}$ Similar to previous studies, all-cause hospitalization using a $0-42$ day window was also evaluated.., 19

\section{Covariates}

Covariates assessed included age, gender, race/ethnicity, Medicaid insurance at least one month in the previous year, neighborhood poverty, and neighborhood rates of asthma, classified based on rates of asthma hospital admission by ZIP code data from the Minnesota Department of Health. ${ }^{20}$ 
Algorithms were developed and validated to classify subjects by asthma control (controlled versus not controlled) and by asthma severity (persistent or intermittent) based on chart abstraction data from the 12 months prior to immunization. ${ }^{16}$ Asthma control corresponded to children with a) one or no prescriptions or fills for an oral corticosteroid, and b) three or fewer prescriptions or fills for albuterol, and c) one or no emergency department visits for asthma, and d) no hospitalizations for asthma. Subjects not meeting all of four criteria were classified as having uncontrolled asthma. Persistent asthma was defined as having one or more prescriptions or fills for an inhaled or nebulized steroid in previous year, and if not it was defined as intermittent asthma. These algorithms were developed following the work by Birnbaum $^{21}$ and Leidy. ${ }^{22}$

\section{Analysis}

Baseline characteristics of subjects were compared by medical group for the periods preand post-guideline. Using a pre/post LAIV guideline/control ratio of ratios (ROR), we evaluated 21 and 42 day rates for LRE following vaccination. The ratio of pre and post LRE rates in LAIV guideline group was compared to those in the control group. Asthma severity, asthma control, age, race/ethnicity, and on Medicaid were included as covariates in the final models. Analysis using an ROR approach limits potential confounding between groups, and accounts for secular trends. To estimate the parameters of interest, we used the generalized estimating equation regression model with Poisson distribution and log link, and a compound symmetry covariance to account for multiple seasons per children. The main parameter of interest was the period- medical group guideline interaction. Rates for LRE following vaccination were calculated for 0-21 and 0-42 day risk windows for each period and medical group. Hospitalization rates were similarly reported using a 42-day risk window. The ratio of ratios (interaction term) with $95 \%$ confidence interval was used to evaluate the impact of the LAIV guideline. Associations were evaluated using a p-value of 0.05 with two tails. We also conducted a sensitivity analysis to model the effect of residual unmeasured confounders. ${ }^{23}$ Our study had $80 \%$ power to detect a ROR of 1.7 with a baseline 42 day LRE rate of 4 percent, with an alpha of 0.05. All analyses were conducted using SAS 9.4 software (SAS Institute Inc., Cary, NC, USA).

\section{Results}

We identified 20,099 children with asthma ages 2 through 17 years, corresponding to 43,298 influenza vaccinations from the 2007-08 through the 2014-15 influenza seasons. We excluded a total of 35,447 child-influenza records; 30,704 because of not meeting minimum insurance enrollment, 150 having a contraindication to receiving LAIV, 246 with an inpatient or ER encounter within 42 days prior to vaccination, and 4,347 with outpatient LRE in the 42 days before vaccination (Figure 1). A total of 4771 unique children and 7851 child-influenza records were included in the cohort. During the three pre-guideline influenza seasons (2007-08, 2008-09, 2009-10) there were 2215 child-influenza records from children and adolescents included from the LAIV group and 735 from the IIV guideline group. For the five post-guideline influenza seasons (2010-11, 2011-12, 2012-13, 2013-14, 2014-15) there were 3767 child-influenza records in children and adolescents from the LAIV group and 1134 from the IIV guideline group. The population receiving care at the 
LAIV group was more likely than the population receiving care at the IIV group to be African American (23\% versus 15\%), and public insurance use were higher in the LAIV guideline group during the post-guideline period (Table 1). In both medical groups and both time periods, roughly half of the children and adolescents had intermittent asthma while half had persistent asthma. About $81 \%$ of the LAIV group children and $76 \%$ of the IIV group children had asthma under control. In the LAIV group, $23 \%$ of subjects included in the cohort with asthma or recurrent wheezing received LAIV in the pre-guideline period, and this proportion increased to $68 \%$ in the post-guideline period. In the IIV group, the proportion of patients in our cohort with asthma or recurrent wheezing who received LAIV were $7 \%$ and $11 \%$ during these two periods.

Overall, $88 \%$ of LREs included diagnoses for asthma exacerbations as defined by our algorithm. In age, asthma severity, asthma control, race ethnicity and Medicaid adjusted analyses there was no increase in LREs associated with the LAIV guideline: overall adjusted (aROR) (95\%CI): $0.74(0.43-1.29)$ for LRE within 21 days of vaccination and 0.77 (0.531.14) for LRE within 42 days of vaccination. Results were similar when stratified by age group; for ages 2 through 4 years aROR: 0.92 (0.34-2.53) for LRE within 21 days of vaccination and $0.94(0.49-1.82)$ for LRE within 42 days of vaccination. For ages 5-18 years aROR (95\% CI): $0.58(0.26-1.30)$ for LRE within 21 days of vaccination and 0.67 (0.37-1.23) for LRE within 42 days of vaccination. Inclusion of additional covariates did not modify the ROR estimates.

Only 21 hospitalizations occurred within 42 days of influenza vaccination, and risks for hospitalization were not increased with the LAIV guideline aROR1.47 (0.13-17.2).

\section{Discussion}

This study took advantage of a unique natural experiment to evaluate the safety of a clinical guideline recommending LAIV for children and adolescents with asthma or recurrent wheezing, as compared with a standard guideline recommending IIV for this population. After adjusting for age, asthma severity and asthma control, the increase of LAIV use from $23 \%$ to $68 \%$ in children with asthma in the LAIV group compared to an increase of LAIV use of only from $7 \%$ to $11 \%$ in the control group was not associated with an increase in LRE, primarily asthma or wheezing, in 21 days or 42 days post vaccination, including in the youngest subgroup of children (those 2 through 4 years of age).There is little evidence for vaccine induced wheezing in children with asthma over age 2. In 2003 in a randomized controlled trial of LAIV versus placebo, Bergen et al found a significant increase in asthma exacerbations among 18-36 month olds within 42 days of vaccination (OR 4.06). ${ }^{9}$ However, in Bergen's study there was no time clustering of events following vaccination and when combining the diagnoses of asthma, shortness of breath and wheezing, differences between LAIV and placebo were no longer significant. Bergen did a post-hoc analysis evaluating children with a previous history of asthma or reactive airway disease, and no increased risk following LAIV was detected (RR 1.11). In a subsequent 2007 multisite randomized controlled trial, Belshe reported increased wheezing in children ages 6 to 11 months and increased all-cause hospitalization for children younger than 2 years following LAIV versus IIV. However, no increase in wheezing occurred in children over age 12 months. ${ }^{5}$ Using 
spirometry, Redding demonstrated no change in pulmonary function following LAIV among children with asthma 9 years and older. ${ }^{11}$

Findings from this study are consistent with several recent observational studies of LAIV in children and adolescents with asthma. Ray et al. found that among children $\geq 2$ years old with asthma, there was no increased risk of asthma exacerbation following LAIV or IIV and a decreased risk following LAIV compared to IIV. The children given LAIV had less severe asthma and this likely biased the outcome. ${ }^{12}$ Duffy et al. found that LAIV use in asthma was associated with a decreased risk of medically attended respiratory adverse events, but that the LAIV vaccine was used primarily in children with intermittent or mild persistent asthma. ${ }^{13}$ Studies of Vaccine Adverse Events System reports found no increase in adverse events, including wheezing, after trivalent and quadrivalent LAIV. ${ }^{24}$

Currently in the United States, asthma at any age and recurrent wheezing in children younger than 5 years are both precautions for administering LAIV. ${ }^{8}$ However, international policies regarding the use of LAIV in children with asthma vary. Starting in the 2012-13 influenza season, Quebec recommended LAIV for children with asthma and other chronic health conditions. ${ }^{25}$ Similarly, the United Kingdom's National Health Service now recommends LAIV for all children, including those with asthma. ${ }^{26}$ Data on the safety of Quebec's and the United Kingdom's policies have yet to be reported.

This study had some limitations. First, we were limited to data available in the EHR or claims records. As such our identification of patients with asthma and events to be classified as a LRE were limited to those identifiable through diagnostic codes and we were only able to capture medically attended LREs. We were not able to evaluate episodes of wheezing that may have been treated at home. Furthermore, we did not evaluate upper respiratory events that may occur following LAIV, such as nasal discharge or sinusitis. Nevertheless, our findings are reassuring for ruling out risks for severe respiratory events following adoption of an LAIV guideline. Second, exclusion of asthma management encounters and classification of asthma severity were based on diagnoses, visits, and medication orders and fills. As such, these estimates are prone to misclassification which would have biased our findings to the null. Third, several important covariates were not available including daycare attendance, presence of school-age siblings, and exposure to second-hand smoke. Nevertheless, our pre-post intervention controlled design removes bias in the association measure due to multiple causes, including unobserved time varying factors that could affect the outcomes in both groups in similar ways. ${ }^{14}$

The study also had several strengths including the large number of children and adolescents with asthma receiving influenza vaccination and the analytic approach using ROR. Also, $41 \%$ of the LAIV post-guideline group was classified as having persistent asthma and 19\% as having uncontrolled asthma. As such, our results are less prone to confounding by indication compared to studies where only patients with mild intermittent asthma would be offered LAIV. 


\section{Conclusion}

In this large observational study, a guideline recommending LAIV for children and adolescents with asthma or recurrent wheezing was found not to increase the risk of postvaccination medically attended LREs. These additional data strengthen existing data suggesting that the LAIV vaccine is safe for children with asthma older than 2 years of age, and that guidelines for influenza vaccination in children with asthma over age 2 should allow use of either vaccine and be based on vaccine effectiveness.

\section{Acknowledgments}

Funding: This study was funded by National Institute of Allergy and Infectious Diseases/NIH/DHHS, Grant R21AI122141

\section{Abbreviations}

LAIV

IIV

LRE

ROR

aROR

ACIP

ICD-9-CM

ED

$\mathbf{R R}$ live attenuated influenza vaccine

inactivated influenza vaccine

lower respiratory event

ratio of rate ratio (analysis)

adjusted ratio of rate ratio

Advisory Committee on Immunization Practices

International Classification of Diseases-9-clinical modification

emergency department

relative risk

\section{References}

1. Nichol KL. Cost-effectiveness and socio-economic aspects of childhood influenza vaccination. Vaccine. 2011;29(43):7554-7558. [PubMed: 21820477]

2. Dawood FS, Kamimoto L, D'Mello TA, et al. Children with asthma hospitalized with seasonal or pandemic influenza, 2003-2009. Pediatrics. 2011;128(1):e27-32. [PubMed: 21646257]

3. Burney LE. Influenza immunization. The Journal of the Oklahoma State Medical Association. 1960;53:830-833. [PubMed: 13689173]

4. Fiore AE, Uyeki TM, Broder K, et al. Prevention and control of influenza with vaccines: recommendations of the Advisory Committee on Immunization Practices (ACIP), 2010. MMWR Recomm Rep. 2010;59(RR-8):1-62.

5. Belshe RB, Edwards KM, Vesikari T, et al. Live attenuated versus inactivated influenza vaccine in infants and young children. N Engl J Med. 2007;356(7):685-696. [PubMed: 17301299]

6. Ashkenazi S, Vertruyen A, Aristegui J, et al. Superior relative efficacy of live attenuated influenza vaccine compared with inactivated influenza vaccine in young children with recurrent respiratory tract infections. Pediatr Infect Dis J. 2006;25(10):870-879. [PubMed: 17006279]

7. Fleming DM, Crovari P, Wahn U, et al. Comparison of the efficacy and safety of live attenuated cold-adapted influenza vaccine, trivalent, with trivalent inactivated influenza virus vaccine in children and adolescents with asthma. Pediatr Infect Dis J. 2006;25(10):860-869. [PubMed: 17006278] 
8. Grohskopf LA, Sokolow LZ, Fry AM, Walter EB, Jernigan DB. Update: ACIP Recommendations for the Use of Quadrivalent Live Attenuated Influenza Vaccine (LAIV4) - United States, 2018-19 Influenza Season. MMWR Morb Mortal Wkly Rep. 2018;67(22):643-645. [PubMed: 29879095]

9. Bergen R, Black S, Shinefield H, et al. Safety of cold-adapted live attenuated influenza vaccine in a large cohort of children and adolescents. Pediatr Infect Dis J. 2004;23(2):138-144. [PubMed: 14872180]

10. Piedra PA, Gaglani MJ, Riggs M, et al. Live attenuated influenza vaccine, trivalent, is safe in healthy children 18 months to 4 years, 5 to 9 years, and 10 to 18 years of age in a communitybased, nonrandomized, open-label trial. Pediatrics. 2005;116(3):e397-407. [PubMed: 16140685]

11. Redding G, Walker RE, Hessel C, et al. Safety and tolerability of cold-adapted influenza virus vaccine in children and adolescents with asthma. Pediatr Infect Dis J. 2002;21(1):44-48. [PubMed: 11791098]

12. Ray GT, Lewis N, Goddard K, et al. Asthma exacerbations among asthmatic children receiving live attenuated versus inactivated influenza vaccines. Vaccine. 2017;35(20):2668-2675. [PubMed: 28404355]

13. Duffy J, Lewis M, Harrington T, et al. Live attenuated influenza vaccine use and safety in children and adults with asthma. Annals of allergy, asthma \& immunology : official publication of the American College of Allergy, Asthma, \& Immunology. 2017;118(4):439-444.

14. Barnighausen T, Rottingen JA, Rockers P, Shemilt I, Tugwell P. Quasi-experimental study designs series-paper 1: introduction: two historical lineages. J Clin Epidemiol. 2017;89:4-11. [PubMed: 28694121]

15. Fiore AE, Shay DK, Broder K, et al. Prevention and control of influenza: recommendations of the Advisory Committee on Immunization Practices (ACIP), 2008. MMWR Recomm Rep. 2008;57(RR-7):1-60.

16. Nordin JD, Vazquez Benitez G, Kharbanda EO, Olsen A, Kuckler L. Identifying Asthma Severity and Visits for Asthma Exacerbations From Chart Audit Data. 24th Annual Health Care Systems Research Network Conference; 2018; Minneapolis, Minnesota.

17. Baxter R, Toback SL, Sifakis F, et al. A postmarketing evaluation of the safety of Ann Arbor strain live attenuated influenza vaccine in adults 18-49 years of age. Vaccine. 2012;30(20):3053-3060. [PubMed: 22425787]

18. Toback SL, Ambrose CS, Eaton A, et al. A postlicensure evaluation of the safety of Ann Arbor strain live attenuated influenza vaccine in children 24-59 months of age. Vaccine. 2013;31(14): 1812-1818. [PubMed: 23395734]

19. Prosser LA, Meltzer MI, Fiore A, et al. Effects of Adverse Events on the Projected Population Benefits and Cost-effectiveness of Using Live Attenuated Influenza Vaccine in Children Aged 6 Months to 4 Years. Arch Pediatr Adolesc Med. 2011;165(2):112-118. [PubMed: 20921341]

20. MDH. MN Public Health Data Access. Asthma Hospitalizations: All ages, 2007-2011. 2014; https://apps.health.state.mn.us/mndata/asthmazip_map. Accessed February 3, 2015.

21. Birnbaum HG, Ivanova JI, Yu AP, et al. Asthma severity categorization using a claims-based algorithm or pulmonary function testing. The Journal of asthma : official journal of the Association for the Care of Asthma. 2009;46(1):67-72. [PubMed: 19191141]

22. Leidy KN, Paramore LC, Watrous M, Doyle J, Zeiger RS. Development of an algorithm for estimating asthma severity from an administrative cost database. Value Health 1999; 2:394DIS 2005, Vol 2 Technical SpecificationsWashington, DC. 1999.

23. Groenwold RH, Nelson DB, Nichol KL, Hoes AW, Hak E. Sensitivity analyses to estimate the potential impact of unmeasured confounding in causal research. Int J Epidemiol. 2010;39(1):107117. [PubMed: 19948779]

24. Haber P, Moro PL, Cano M, Lewis P, Stewart B, Shimabukuro TT. Post-licensure surveillance of quadrivalent live attenuated influenza vaccine United States, Vaccine Adverse Event Reporting System (VAERS), July 2013-June 2014. Vaccine. 2015;33(16):1987-1992. [PubMed: 25678241]

25. Quach C Vaccinating high-risk children with the intranasal live-attenuated influenza vaccine: the Quebec experience. Paediatric respiratory reviews. 2014;15(4):340-347. [PubMed: 25242731] 
26. NHS. The Green Book, Influenza, Chapter 19, Updated December 2014 2014; https://www.gov.uk/ government/uploads/system/uploads/attachment_data/file/385226/

Green_Book_Chapter_19_v8_2.pdf. Accessed February 9, 2014. 


\section{Highlights}

Two early trials of LAIV found subsequent increased wheezing in young children.

Little data exists on wheezing after LAIV in children with asthma.

In this study, 7851 doses of influenza vaccine were given to children with asthma.

No increased wheezing occurred with a guideline recommending LAIV for children with asthma.

We conclude an LAIV guideline is safe in children age 2 to 17 years with asthma. 


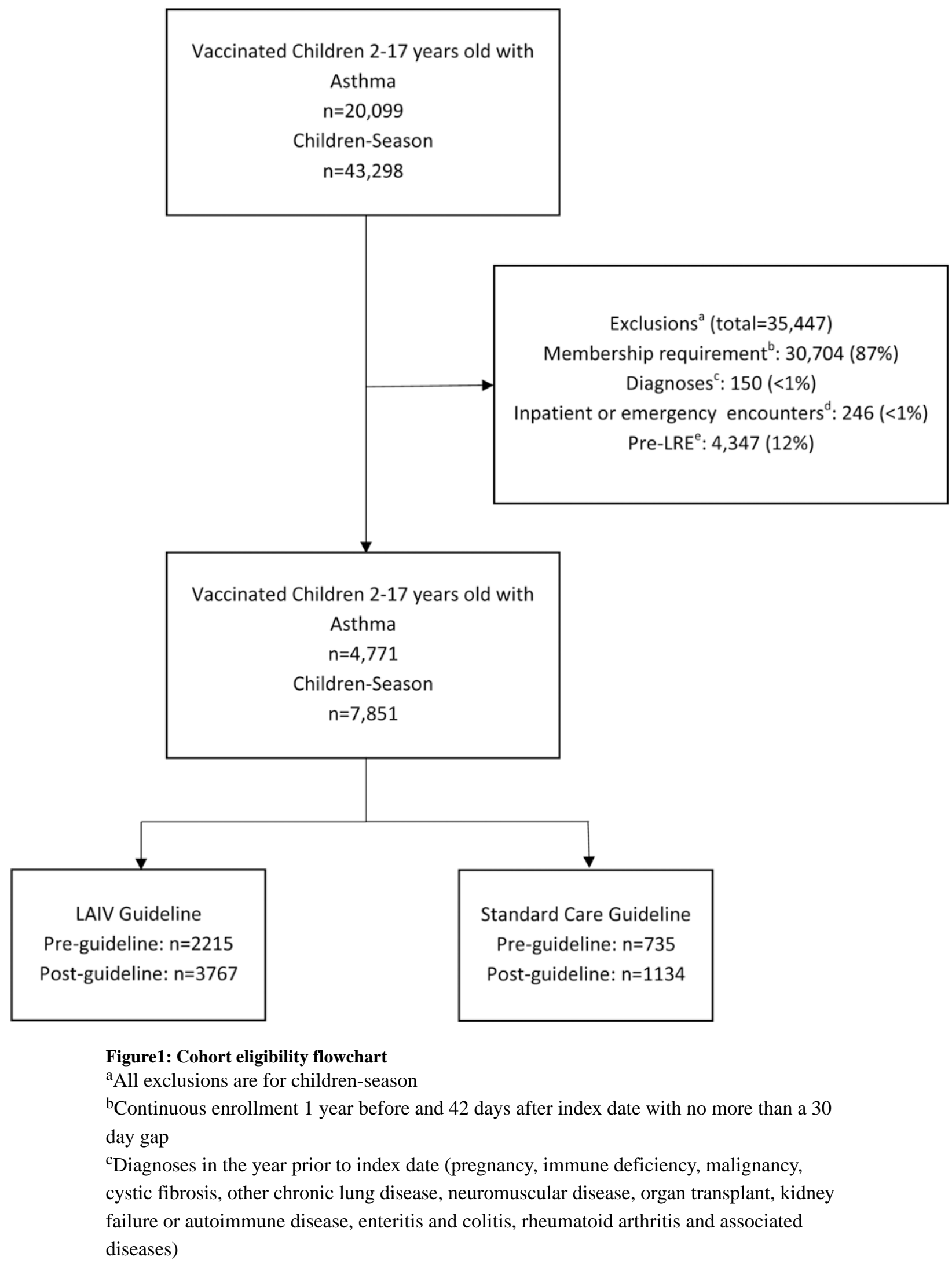

Vaccine. Author manuscript; available in PMC 2020 July 09. 
${ }^{\mathrm{d}}$ Those with an inpatient or emergency encounter in the 42 days before the index date ${ }^{\mathrm{e}}$ Those with an outpatient visit for a lower respiratory event (LRE) in the 42 days before the index date 
Table 1.

Characteristics of children with asthma, Pre- and Post-Guideline by medical group, $n=4771$ unique children $^{a}$

\begin{tabular}{|c|c|c|c|c|}
\hline & \multicolumn{2}{|c|}{$\begin{array}{l}\text { LAIV Guideline medical } \\
\text { group }\end{array}$} & \multicolumn{2}{|c|}{ Standard care medical grour } \\
\hline & $\begin{array}{c}\text { Pre- } \\
\text { Guideline }\end{array}$ & $\begin{array}{l}\text { Post- } \\
\text { Guideline }\end{array}$ & $\begin{array}{c}\text { Pre- } \\
\text { Guideline }\end{array}$ & $\begin{array}{l}\text { Post- } \\
\text { Guideline }\end{array}$ \\
\hline \multirow[t]{5}{*}{ Influenza seasons } & $2007-08$ & $2010-11$ & $2007-08$ & $2010-11$ \\
\hline & 2008-09 & 2011-12 & 2008-09 & 2011-12 \\
\hline & $2009-10$ & $2012-13$ & $2009-10$ & $2012-13$ \\
\hline & & 2013-14 & & 2013-14 \\
\hline & & $2014-15$ & & $2014-15$ \\
\hline Children in each group & 1641 & 2515 & 552 & 786 \\
\hline Female & $664(40.5)$ & $1058(42.1)$ & $241(43.7)$ & $313(39.8)$ \\
\hline Race / ethnicity $\quad \mathrm{n}(\%)$ & & & & \\
\hline Asian & $129(7.9)$ & $236(9.4)$ & $45(8.2)$ & $59(7.5)$ \\
\hline Black/African American & $367(22.4)$ & $609(24.2)$ & $85(15.4)$ & $114(14.5)$ \\
\hline Hispanic & $101(6.2)$ & $153(6.1)$ & $26(4.7)$ & $34(4.3)$ \\
\hline Other & $72(4.4)$ & $108(4.3)$ & $30(5.4)$ & $32(4.1)$ \\
\hline White & $972(59.2)$ & $1409(56)$ & $366(66.3)$ & 547 (69.6) \\
\hline Use of Interpreter $\mathrm{n}(\%)$ & $21(1.3)$ & $55(2.2)$ & $12(2.2)$ & $11(1.4)$ \\
\hline Medicaid & $328(20)$ & $739(29.4)$ & $103(18.7)$ & $143(18.2)$ \\
\hline
\end{tabular}

${ }^{a}$ Children counted once per period of care and per medical group 
Table 2.

Characteristics of children-seasons included in cohort, Pre- and Post-Guideline by medical group, $\mathrm{n}=7851$ child-seasons $^{a}$

\begin{tabular}{|c|c|c|c|c|}
\hline & \multicolumn{2}{|c|}{$\begin{array}{c}\text { LAIV Guideline medical } \\
\text { group }\end{array}$} & \multicolumn{2}{l|}{ Standard care medical group } \\
\hline & $\begin{array}{c}\text { Pre- } \\
\text { Guideline }\end{array}$ & $\begin{array}{c}\text { Post- } \\
\text { Guideline }\end{array}$ & $\begin{array}{c}\text { Pre- } \\
\text { Guideline }\end{array}$ & $\begin{array}{c}\text { Post- } \\
\text { Guideline }\end{array}$ \\
\hline Child-seasons & 2215 & 3767 & 735 & 1134 \\
\hline Age groups (in years) & & & & \\
\hline $2-4 \mathrm{n}(\%)$ & $631(28.5)$ & $1059(28.1)$ & $265(36.1)$ & $287(25.3)$ \\
\hline $5-11 \mathrm{n}(\%)$ & $868(39.2)$ & $1539(40.9)$ & $265(36.1)$ & $495(43.7)$ \\
\hline $12-17 \mathrm{n}(\%)$ & $716(32.3)$ & $1169(31)$ & $205(27.9)$ & $352(31)$ \\
\hline Received LAIV n (\%) & $517(23.3)$ & $2542(67.5)$ & $50(6.8)$ & $127(11.2)$ \\
\hline Mean asthma hospital admission rate per 10,000 in neighborhood +SD & $32.79+20.17$ & $34.19+21.07$ & $29.68+22.15$ & $30.01+19.97$ \\
\hline Mean Median Family income in neighborhood (1000)+SD & $83.11+30.09$ & $79.11+29.96$ & $96.84+35.5$ & $98.78+35.77$ \\
\hline Preventive Care visit $\mathrm{n}(\%)$ & $1356(61.2)$ & $2301(61.1)$ & $432(58.8)$ & $626(55.2)$ \\
\hline Intermittent Asthma $\mathrm{n}(\%)$ & $1009(45.6)$ & $2027(53.8)$ & $314(42.7$ & $51245.1)$ \\
\hline Controlled Asthma ${ }^{b}$ n (\%) & $1781(80.4)$ & $3057(81.2)$ & $544(74)$ & $886(78.1)$ \\
\hline
\end{tabular}

${ }^{a}$ Children were counted multiple times if received the vaccine in multiple vaccine seasons. Only the first vaccine of the season is counted. Only 72 children had more than one vaccine in the same season, and all were administered more than 42 days apart.

$b$ based on asthma control algorithm (see text) 
Table 3.

Lower Respiratory Event (LRE) rates, n (\%), within 21 and 42 days of influenza vaccination, by guideline with ratio of rate ratios

\begin{tabular}{|c|c|c|c|c|c|c|}
\hline & \multicolumn{2}{|c|}{$\begin{array}{l}\text { LAIV Guideline } \\
\text { medical Group }\end{array}$} & \multicolumn{2}{|c|}{$\begin{array}{l}\text { Standard care } \\
\text { medical group }\end{array}$} & \multirow{2}{*}{$\begin{array}{l}\text { Crude Ratio of } \\
\text { Rate Ratios } \\
(\mathbf{9 5 \%} \text { CI })\end{array}$} & \multirow{2}{*}{$\begin{array}{l}\text { Adjusted Ratio of } \\
\text { Rate Ratios } \\
(95 \% \text { CI) }\end{array}$} \\
\hline & $\begin{array}{l}\text { Pre- } \\
\text { guideline } \\
\text { n }(\%)\end{array}$ & $\begin{array}{l}\text { Post- } \\
\text { guideline } \\
\text { n }(\%)\end{array}$ & $\begin{array}{l}\text { Pre- } \\
\text { guideline } \\
\text { n }(\%)\end{array}$ & $\begin{array}{l}\text { Post- } \\
\text { guideline } \\
\text { n }(\%)\end{array}$ & & \\
\hline \multicolumn{7}{|l|}{ Children age 2 to 17 years } \\
\hline LRE in 21 days & $77(3.5)$ & $113(3.0)$ & $27(3.7)$ & $45(4.0)$ & $0.80(0.46-1.39)$ & $0.74(0.43-1.29)$ \\
\hline LRE in 42 days & $133(6)$ & $203(5.4)$ & $51(6.9)$ & $85(7.5)$ & $0.83(0.56-1.22)$ & $0.77(0.53-1.14)$ \\
\hline All Cause Hospitalizations & $12(0.5)$ & $7(0.2)$ & $3(0.4)$ & $1(0.1)$ & $1.59(0.14-18.30)$ & $1.47(0.13-17.2)$ \\
\hline \multicolumn{7}{|l|}{ Children age $2-4$ years } \\
\hline LRE in 21 days & $26(4.1)$ & $43(4.1)$ & $10(3.8)$ & $11(3.8)$ & $0.97(0.36-2.61)$ & $0.92(0.34-2.53)$ \\
\hline LRE in 42 days & $42(6.7)$ & $84(7.9)$ & $20(7.5)$ & $26(9.1)$ & $0.99(0.51-1.93)$ & $0.94(0.49-1.82)$ \\
\hline \multicolumn{7}{|l|}{ Children age $5-17$ years } \\
\hline LRE in 21 days & $51(3.2)$ & $70(2.6)$ & $17(3.6)$ & $34(4.0)$ & $0.63(0.28-1.41)$ & $0.58(0.26-1.30)$ \\
\hline LRE in 42 days & $91(5.7)$ & $119(4.4)$ & $31(6.6)$ & $59(7.0)$ & $0.69(0.37-1.27)$ & $0.67(0.37-1.23)$ \\
\hline
\end{tabular}

${ }^{1}$ Adjusted for age, asthma severity and control, race/ethnicity and on Medicaid. 